\title{
Éditorial
}

\section{Centenaire de la Revue de Métallurgie}

Dans le numéro de janvier 1984 de La Revue de Métallurgie, Henri Malcor et Jean-Alex Michard, alors Directeur Général de l'Irsid et Président Directeur Général de La Revue de Métallurgie et du CDS, signaient un éditorial qui résumait fort bien les 80 années d'histoire de la Revue et ils nous donnaient rendez-vous 20 ans plus tard pour le centenaire.

Aujourd'hui ces 20 années supplémentaires se sont écoulées et c'est pour moi l'occasion de souligner la contribution essentielle apportée par La Revue de Métallurgie à la diffusion des connaissances techniques et scientifiques dans le domaine des Matériaux et plus particulièrement dans le domaine de la Sidérurgie.

Henry Le Chatelier qui créa La Revue de Métallurgie en 1904 a joué un rôle déterminant dans le développement de la science métallurgique au début du XXe siècle. II a en effet publié dans la Revue un mémoire dans chaque numéro mensuel pendant les premières années et contribué à la diffusion des idées de $W$. Taylor dans le domaine de l'organisation scientifique du travail.

Par la suite, de nombreux savants, chercheurs et ingénieurs ont choisi La Revue de Métallurgie pour publier leurs travaux comme le rappelle le premier article de ce numéro du centenaire : "La Revue de Métallurgie, cent ans d'histoire ».

Science et Industrie : d'emblée H. Le Chatelier a cherché à réconcilier les 2 sœurs jumelles et faire en sorte que leur interpénétration réciproque soit source de nouveaux progrès. L'histoire de la Revue est ainsi faite de séparations et de rapprochements entre les 2 sœurs ; séparation en 1959 avec la création des Mémoires Scientifiques distincts de la Revue elle-même, rapprochement en 1993 avec la fusion de la Revue de Métallurgie-Cahiers d'Informations Techniques avec les Mémoires Scientifiques.

Ces mouvements d'aller et retour traduisent en réalité deux difficultés :

La première concerne l'intérêt du lectorat ; même s'il est souhaitable de faire partager au plus grand nombre la diversité des approches techniques et scientifiques, le chercheur est davantage intéressé par la modélisation et la théorie des phénomènes alors que l'ingénieur est plus concerné par l'analyse des évènements et leurs conséquences. Chacun doit faire un effort pour être compris de l'autre alors qu'il est plus facile et plus rapide de dialoguer avec ses pairs.

La deuxième est d'ordre économique et est sous-tendue par le fait qu'une revue de qualité ne peut être viable financièrement que si le nombre de lecteurs potentiels est suffisant. Or chacun connaît l'érosion de nos effectifs industriels mais aussi la diminution du nombre de chercheurs et universitaires impliqués dans le domaine des matériaux.

Par ailleurs les rapprochements entre Sociétés Sidérurgiques et entre Universités et Centres de Recherche se multiplient en Europe justifiant ainsi le besoin de revues scientifiques et techniques ayant une forte assise européenne. Je sais que la Revue de Métallurgie a entamé des discussions avec d'autres publications dans le but de jeter les bases d'une Revue Technique et de Revues Scientifiques européennes de qualité et j'encourage donc vivement la Revue de Métallurgie à poursuivre dans cette direction ; c'est la meilleure façon de se donner une nouvelle jeunesse et de prendre un bon départ pour atteindre le deuxième centenaire. 
Le numéro spécial préparé pour le premier centenaire de la Revue de Métallurgie est en tout point remarquable par la qualité et la diversité des thèmes abordés qui couvrent un très large éventail de la technique et de la science métallurgique. Je voudrais remercier tout particulièrement le professeur Friedel, membre de l'Académie des Sciences, qui s'appuyant sur son incomparable expérience personnelle analyse dans son article " sur l'emploi de modèles en métallurgie ", la nécessité et les limites des modèles théoriques pour conduire les progrès en métallurgie.

Je suis certain que ce numéro du centenaire restera longtemps un ouvrage de référence pour les métallurgistes et j'encourage tous les chercheurs et tous les ingénieurs à publier et faire connaître leurs travaux dans les revues techniques et scientifiques qui restent un maillon indispensable de la chaîne complexe qui conduit au progrès.

Bonne lecture à tous.

\section{Guy DOLLÉ}

Président de la Direction Générale d'Arcelor

Président de la Fédération Française de l'Acier 